Received: 2016.12 .20

Accepted: 2017.03 .03

Published: 2017.12 .15

Authors' Contribution:
A Study Design
B Data Collection
C Statistical Analysis
D Data Interpretation
E Manuscript Preparation
F Literature Search
G Funds Collection

\section{Evaluation of Alterations in Tumor Tissue of Gastrointestinal Stromal Tumor (GIST) in Computed Tomography Following Treatment with Imatinib}

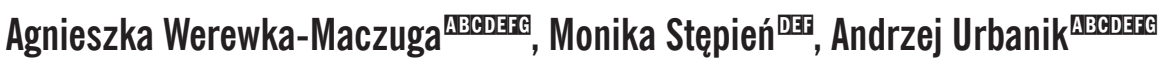

Department of Radiology, Jagiellonian University Medical College, Cracow, Poland

Author's address: Monika Stępień, Department of Radiology, Jagiellonian University Medical College, Kopernika 19 Str., 31-501 Cracow, Poland, e-mail: mdmonikas@gmail.com

Background: The aim of this study was to evaluate the alterations in the neoplastic tissue of GIST following Imatinib treatment.

\begin{abstract}
Material/Methods:
CT studies of 14 patients with inoperable primary tumors and 56 patients with metastatic and recurrent disease after chemotherapy were analyzed retrospectively. The following alterations in features of primary and secondary tumors were analyzed: dimension, degree and type of contrast enhancement, outlines of lesions, presence of intratumoral bleeding, presence of calcifications.

Results: In the analyzed group of primary, metastatic and recurrent tumors after treatment with Imatinib in most cases a decrease in size and contrast enhancement were observed; the outlines of lesions became well circumscribed. Following the treatment, the number of tumors enhancing inhomogeneously decreased. In primary tumors the percentage of calcifications increased, whereas in metastatic tumors calcifications were observed only after treatment. There was no bleeding found within primary tumors after treatment. In metastatic disease, increased percentage of tumors with transient intratumoral bleeding was observed. There were also some unconventional CT images following treatment, such as: cystic transformation of lesions, enlargement of lesions, appearing of new lesions suggesting progression of the disease, stationary dimensions of lesions during local progression of the disease, simultaneous decrease and increase in size of metastatic lesions or appearance of new ones.
\end{abstract}

Conclusions: $\quad$ Right from the start of Imatinib therapy in inoperable and disseminated GIST patients, specific CT images, not seen during conventional cytotoxic chemotherapy, were observed.

MeSH Keywords: $\quad$ Drug Therapy • Gastrointestinal Stromal Tumors • Multidetector Computed Tomography

PDF file: $\quad$ http://www.polradiol.com/abstract/index/idArt/902944

\section{Background}

Gastrointestinal stromal tumor (GIST) is the most common gastrointestinal tumor of mesenchymal origin. GIST may develop in every part of the gastrointestinal tract, but mostly occurs in the stomach (50-60\% of cases) and in the small intestine (30-35\% of cases). Less often it occurs in the colon and rectum ( $5 \%$ of cases), and in the esophagus $(<1 \%$ of cases) [1]. In less than 5\% of cases GISTs arise from the omentum, mesentery and retroperitoneal space [2].
Until recently, the only treatment method of GIST was radical surgical resection of the primary lesion. In turn, the treatment effects of inoperable or disseminated GIST were very bad (median overall survival 9 to 20 months after disease recurrence). In recent years, the breakthrough occurred, thanks to introduction of new treatment with Imatinib Mesylate (commercial name Glivec) [3-5]. Imatinib is a phenylaminopyrimidine derivative, its mechanism of action depends on blocking of ATP binding by tyrosine kinase. This action is breaking the signal cascade of the main pathway stimulating the tumor growth. Imatinib is a selective inhibitor of three tyrosine 
kinases: the Bcr kinase, c-KIT kinase and the PDGFRA kinase. Thanks to the discovery of crucial impact of c-KIT and PDGFRA receptor activation on development of GIST, Imatinib-based causal treatment could be applied. This therapy was earlier successfully used against chronic myeloid leukemia. Therefore, GIST became the first solid tumor treated effectively with targeted molecular therapy. The first use of Imatinib took place in 2001. After a series of clinical trials, the drug was allowed for sale in 2002, as a first-line treatment of advanced GIST. Imatinib is a representative of a modern group of anti-tumor cytostatic drugs. It is not a conventional cytostatic drug (the one that is used in wide spectrum of neoplasms and has significant side effects), it is targeted against a specific tumor type, bearing much smaller general toxicity. The benefits of treatment with Imatinib in advanced stages of GIST are unquestionable. The rate of positive responses to therapy ranges from 70 to $80 \%$. Partial response (PR) is the most common - more than $50 \%$ of patients, and stable disease (SD) occurs in $30 \%$. Complete remission occurs only in sporadic cases (about $3-6 \%)$, which is due to drug's mechanism of action that does not cause direct cell death.

Treatment response assessment in GIST tumors during molecular targeted therapy is the new problem in radiology, because of the fact that atypical radiological images may occur, unprecedented so far in conventional cytostatic therapies. Generating of these images is caused by different mechanisms of action of drugs and developing secondary resistance. Their improper interpretation, caused by rigid application of treatment response criteria based on changes of the tumor size, can result in drug withdrawal and preterm patient's death.

It has been shown in recent years, that traditional RECIST and WHO criteria, based just on changes of the tumor size, are sometimes inadequate in assessment of effectiveness of modern molecular-based targeted therapy. Changes in blood supply and metabolism of neoplastic tissue after treatment, reflected by contrast enhancement in computed tomography, can significantly precede changes of the tumor size. Often enough a good response to chemotherapy is only visible as decrease in tumor enhancement without reduction of lesion diameter. Application of the RECIST criteria may lower treatment response rates and in consequence lead to withdrawal of the drug in patients who benefit from the therapy. After the criteria assessing therapyinduced tumor response rates were assessed as inadequate, the Choi classification was introduced. This classification, apart from changes in the tumor diameter, includes also changes in attenuation in the venous phase of dynamic CT scan.

\section{Aim of the study}

The goal of the study was to evaluate in CT alterations in neoplastic tissues in the course of GIST in the course of Imatinib therapy.

\section{Material and Methods}

Retrospective analysis of changes that occurred in tissues of primary tumors and metastases due to treatment with
Imatinib. In the whole analyzed group, there were available CT scans of 14 patients with inoperable primary tumors during chemotherapy and studies of metastatic lesions and recurrences in 56 different patients, but in 8 of them there were both metastases and primary lesion in the same time (62 patients in total). There was an equal number of females and males in the analyzed group, with a median age of 61 years. The youngest patient was 14 years old when she got ill - it is an extremely rare case of pediatric GIST; while two oldest patients were 86 years old.

The CT scans were performed at the Department of Imagine Studies, University Hospital in Cracow, using spiral multiple-row CT scanner - Siemens Sensation 16. Patients were prepared by oral administration of contrast medium Urografin 76\%, in amount of $25 \mathrm{~mL}$ per liter of water in two doses: 0.5 liter for night on the day preceding the study and 0.5 liter two hours before the study. Additionally, the patients were given 0.25 liter of solution ( $5 \mathrm{~mL}$ of Urografin per glass of water) right before the scan to contrast the gastrointestinal tract.

The studies were performed with the following parameters:

- layer thickness $5 \mathrm{~mm}$;

- pitch 0.75;

- increment $5 \mathrm{~mm}$.

In every patient 3D reconstructions (2-mm distance between layers) were performed from raw data obtained during acquisition. The data were processed using Syngo CT2006G console.

The imaging was made after intravenous administration of contrast media Omnipaque or Iomeron, concentration 350 $\mathrm{mg} / \mathrm{ml}, \mathrm{l} \mathrm{ml}$ per kilogram of body weight, using an automatic syringe with a flow rate of $2.5 \mathrm{ml} / \mathrm{s}$; in dynamic, triphasic setting:

- before injection of contrast agent - plain scan;

- on arterial phase (after 20-25 seconds after injection);

- on portal venous phase (after 50-60 seconds);

- on delayed phase (after 5-7 minutes of injection of contrast agent)/

Changes of tumors' features in CT that occurred during therapy in the whole observation period were analyzed (median time over two years):

- size (reduction, increase, stationary lesion);

- contrast enhancement level (no enhancement/very low, low/medium, high);

- type of contrast enhancement (heterogeneous or marginal/homogenous/no enhancement);

- lesion contours (well defined/poorly defined);

- presence of bleeding within tumor (yes/no);

- presence of calcifications (yes/no).

\section{Results}

Analysis of alterations visible in CT examination in primary GIST tumors prior and after the therapy are depicted in Tables 1 and 2 . 
Table 1. The alterations in the size and contrast enhancement of the primary GIST following therapy (14 patients).

\begin{tabular}{clccc}
\hline \multicolumn{2}{c}{ Changes in the size and contrast enhancement of the primary tumors following } & \multicolumn{2}{c}{ Quantity } & Percentage [\%] \\
\hline therapy & Shre & Shank & 14 & $100.0 \%$ \\
\hline \multirow{2}{*}{ Contrast enhancement } & Decreased & 12 & $85.7 \%$ \\
\cline { 2 - 4 } & Stationary & 2 & $14 . \%$ \\
\hline
\end{tabular}

Table 2. The alterations in features of the primary GIST following therapy.

\begin{tabular}{|c|c|c|c|c|c|}
\hline \multirow{2}{*}{\multicolumn{2}{|c|}{ Features of the primary GIST tumors }} & \multicolumn{2}{|c|}{ Prior therapy } & \multicolumn{2}{|c|}{ Following therapy } \\
\hline & & $\mathbf{N}$ & $\%$ & $\mathbf{N}$ & $\%$ \\
\hline \multirow{3}{*}{ Enhancement pattern } & Inhomogenous or marginal & 13 & 100.0 & 9 & 64.3 \\
\hline & Homogenous & - & - & 2 & 14.3 \\
\hline & No enhancement & - & - & 3 & 21.4 \\
\hline \multirow{3}{*}{ Level of enhancement } & Very low or low & 1 & 7.1 & 6 & 42.9 \\
\hline & Average or high & 13 & 92.9 & 5 & 35.7 \\
\hline & No enhancement & - & - & 3 & 21.4 \\
\hline \multirow{2}{*}{ Contours } & Well defined & 1 & 7.1 & 3 & 21.4 \\
\hline & Poorly defined & 13 & 92.9 & 11 & 78.6 \\
\hline \multicolumn{2}{|l|}{ Bleeding } & - & - & - & - \\
\hline \multicolumn{2}{|l|}{ Calcifications } & 4 & 28.6 & 5 & 35.7 \\
\hline
\end{tabular}

All the lesions shrank following the treatment. Their contrast enhancement in most of the cases decreased, apart from two tumors in which it remained unchanged.

Prior treatment, the tumors had inhomogeneous contrast enhancement (including peripheral).

Following therapy, $21 \%$ of lesions had no contrast enhancement anymore and number of lesions with inhomogeneous enhancement decreased to $64 \%$.

Two lesions (14.3\%) were enhancing homogenously.

Tumor enhancement level before the treatment was mostly average or high (93\%). One of the lesions was lowenhancing (7\%). Following therapy, average or high contrast enhancement remained in $36 \%$ of lesions, in $43 \%$ became low or very low.

The lesions' contours in $21 \%$ of cases became almost welldefined ( $7 \%$ prior therapy), and in the rest of the cases they were still irregular $79 \%$ (93\% before treatment).

The percentage of calcifications in tumor mass increased from $29 \%$ to $36 \%$ of cases.

There was no bleeding within primary tumor mass neither before nor following therapy.
Figure 1 shows typical alterations in primary GIST image following treatment (after three months of therapy).

To reassume, primary GIST tumors' size decreases following therapy, as well as their contrast enhancement level. After injection of contrast agent, the enhancement pattern becomes more homogenous. Whatsmore, the contours of primary lesions after treatment became more defined, with increased number of calcifications inside them.

Next, the features of metastatic lesions or local recurrence were analysed, before and after treatment. Data are shown in Tables 3 and 4 . In Table 3 we analysed alterations in size, conrast enhancement level and corelations between these variables. In Table 4 we analysed features of GIST metastases following therapy, such as: type and level of contrast enhancement, lesions' contours, presence of bleeding and calcifications, cyst mimicking and tumor isodensity.

Following chemoterapeutic treatment, most of the lesions decreased $(75 \%)$, and in $14 \%$ of cases remained stationary. Only in $11 \%$ of the cases, the size of lesions increased.

In $79 \%$ of cases, contrast enhancement level decreased, in $14 \%$ it remained stationary, and in $7 \%$ increased.

Mostly, metastatic lesions shrank as well as their contrast enhancement deceased (71\%). Less than $4 \%$ of lesions grew and increased contrast enhancement. 


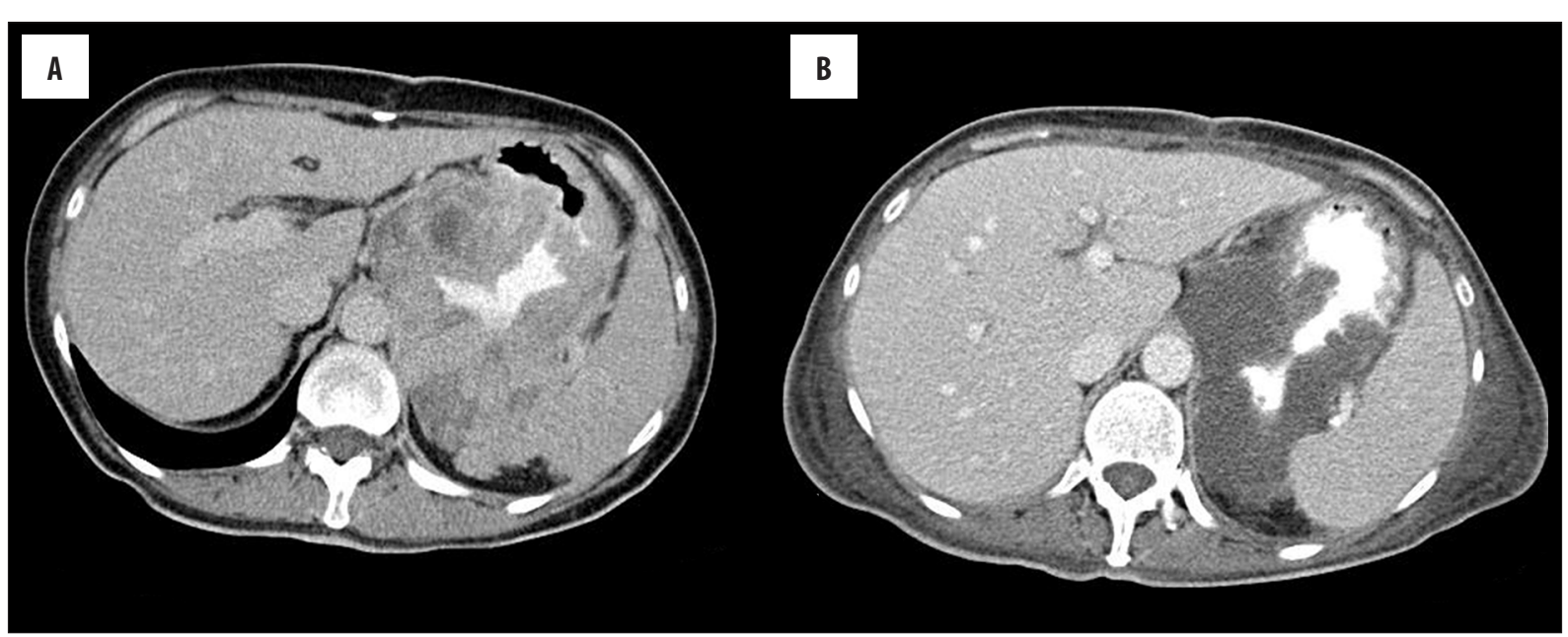

Figure 1. Axial CT scans of GIST in the stomach before (A) and after 3 months (B) of chemotherapy with Imatinib.

Table 3. The relation between the size and contrast enhancement after treatment of the metastases in the course of GIST.

\begin{tabular}{|c|c|c|c|c|c|c|}
\hline \multirow{2}{*}{\multicolumn{3}{|c|}{ Metastases }} & \multicolumn{3}{|c|}{ Size } & \multirow{2}{*}{ Overal } \\
\hline & & & Decreased & Stationary & Increased & \\
\hline \multirow{6}{*}{ Contrast enhancement level } & \multirow{2}{*}{ Decreased } & Quantity & 40 & 2 & 2 & 44 \\
\hline & & Percentage & $71.4 \%$ & $3.6 \%$ & $3.6 \%$ & $78.6 \%$ \\
\hline & \multirow{2}{*}{ Stationary } & Quantity & 2 & 4 & 2 & 8 \\
\hline & & Percentage & $3.6 \%$ & $7.1 \%$ & $3.6 \%$ & $14.3 \%$ \\
\hline & \multirow{2}{*}{ Increased } & Quantity & - & 2 & 2 & 4 \\
\hline & & Percentage & $0 \%$ & $3.6 \%$ & $3.6 \%$ & $7.1 \%$ \\
\hline \multirow{2}{*}{ Overall } & & Quantity & 42 & 8 & 6 & 56 \\
\hline & & Percentage & $75.0 \%$ & $14.3 \%$ & $10.7 \%$ & $100.0 \%$ \\
\hline
\end{tabular}

Table 4. The alterations in features of the metastatic tumors in course of GIST during chemotherapy.

\begin{tabular}{lllccc}
\hline \multirow{2}{*}{ Features of metastases } & \multicolumn{2}{c}{ Before treatment } & \multicolumn{2}{c}{ During treatment } \\
\cline { 2 - 6 } & & $\mathbf{N}$ & $\%$ & N & \% \\
\hline \multirow{3}{*}{ Enhancement type } & Inhomogenous or marginal & 49 & 87.5 & 25 & 44.6 \\
\cline { 2 - 6 } & Homogenous & 7 & 12.5 & 5 & 8.9 \\
\cline { 2 - 6 } & No enhancement & - & - & 26 & 46.4 \\
\hline \multirow{3}{*}{ Enhancement level } & Very low or low & 7 & 12.5 & 15 & 26.8 \\
\cline { 2 - 6 } & Avereage or high & 49 & 87.5 & 15 & 26.8 \\
\cline { 2 - 6 } Contours & No enhancement & - & - & 26 & 46.4 \\
\hline Bleeding & Well defined & 6 & 10.7 & 22 & 39.3 \\
\cline { 2 - 6 } Calcifications & Poorly defined & 50 & 89.3 & 34 & 60.7 \\
\hline Cyst mimicking & & 2 & 3.6 & 7 & 12.5 \\
\hline Isodensity & & 4 & - & 2 & 3.6 \\
\hline
\end{tabular}




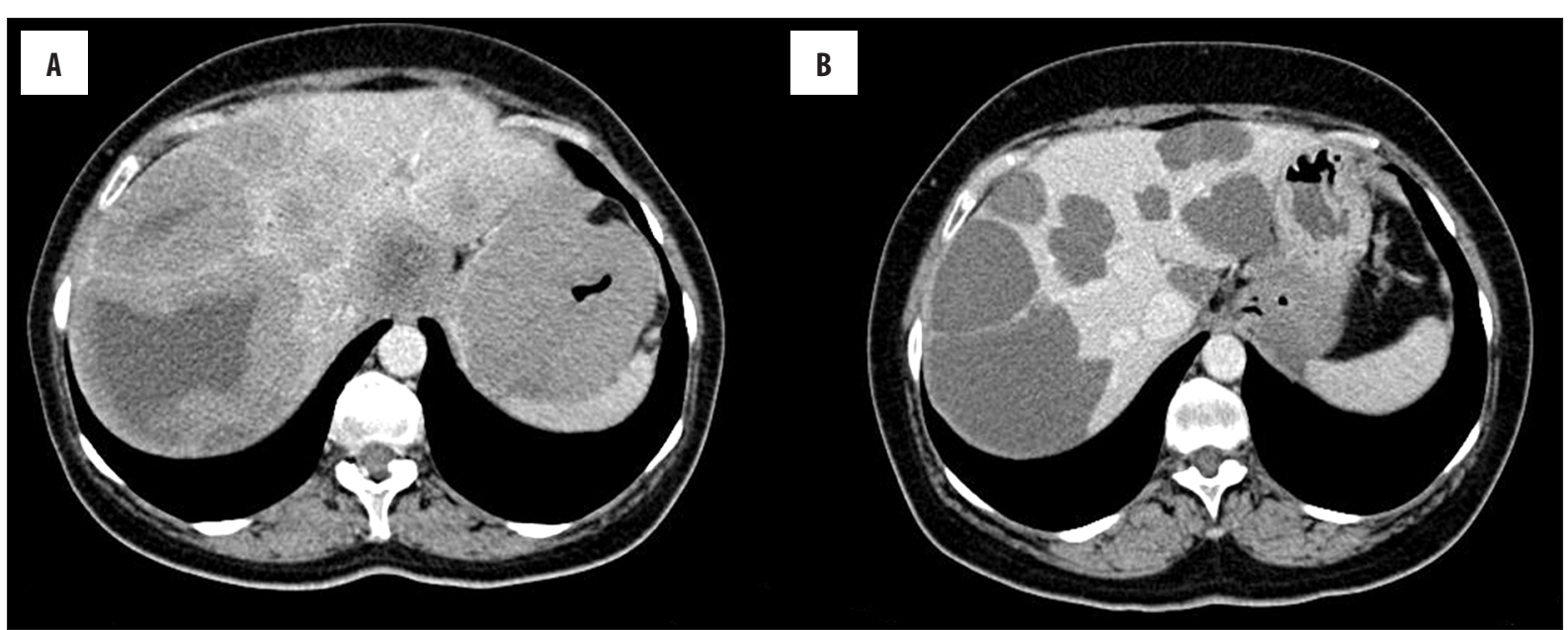

Figure 2. Axial CT scans in the venous phase of the dynamic study of the liver GIST metastases before (A) and after 3 months of chemotherapy (B).

Table 5. The occurrence of the images mimicking progression of lesions in the evaluated group of patients.

\begin{tabular}{lcc}
\hline Pseudoprogression images (with good response to treatment) & Quantity & Percentage [\%] \\
\hline Increase of lesions - size pseudoprogression & 2 & 3.6 \\
\hline Appearance of "new" lesions - number of cases of pseudoprogression & 9 & 16.1 \\
\hline -including both types of pseudoprogression & 1 & \\
\hline No pseudoprogression image & 45 & 80.0 \\
\hline Overall & 56 & 100.0 \\
\hline
\end{tabular}

Before treatment, $87.5 \%$ of lesions were inhomogeneously (including marginally) enhancing, in 12.5\% homogeneously. Following treatment, $46 \%$ of lesions did not show characteristics of contrast enhancement, 54\% still enhanced inhomogeneously (44\%) or homogeneously $(9 \%)$.

Before treatment $87.5 \%$ of lesions enhanced after injection of contrast agent (high and average enhancement level, $12.5 \%$ low or very low). In course of treatment in nearly half of cases, contrast enhancement disappeared after a few months, which was associated with very good response to drug. The remaining tumors were still enhancing low or very low $(27 \%)$, or in average or high level $(27 \%)$.

The contours of lesions in $39 \%$ of cases became welldefined (before treatment only in 11\%).

The features of temporary bleeding into lesions appeared in the course of treatment in $12.5 \%$ of cases ( $4 \%$ before treatment).

Calcifications were visible only in treated metastatic lesions ( $4 \%$ of cases).

Temporarily isodense lesions occurred more often during treatment ( $23 \%$ of cases) than before therapy $(2 \%)$.

After several months of treatment half of the lesions had similar image to cystic changes (50\%), before treatment such an image was sporadic (7\%). Probably that was the reason why in two cases the treated metastases in the liver were described as simple cysts of this organ. There were also descriptions of cysts in the mesentery and fluid collection in the lesser pelvis. In reality those were peritoneal metastases, in which attenuation decreased significantly because of therapy, so they started to look like pseudocysts.

Evaluating changes in attenuation and size of neoplastic lesions in the course of treatment in the whole observation period (median time about 2 years) in the group responding positively to treatment (PR), higher decrease in median attenuation than lesion size was found.

It was noticed that this process occurs most rapidly in the first 3 months after applied treatment.

Figure 2 shows alterations in image of metastatic lesions following Imatinib chemotherapy with development of "pseudocystic" image.

Because of "pseudocyst" phenomenon, CT scans of patients during chemotherapy (62 persons) were analyzed in search of other images of neoplastic pseudoprogression. Data is shown in Table 5. Images mimicking progression consist of appearance of "new" lesions or increasing size of lesions already present despite good response to treatment of neoplastic tissue. In the analysed group of patients decrased attenuation after therapy was considered as a good respose to treatment.

In overall, 11 cases of pseudoprogression were noticed, including its both types in one patient in the same time, 


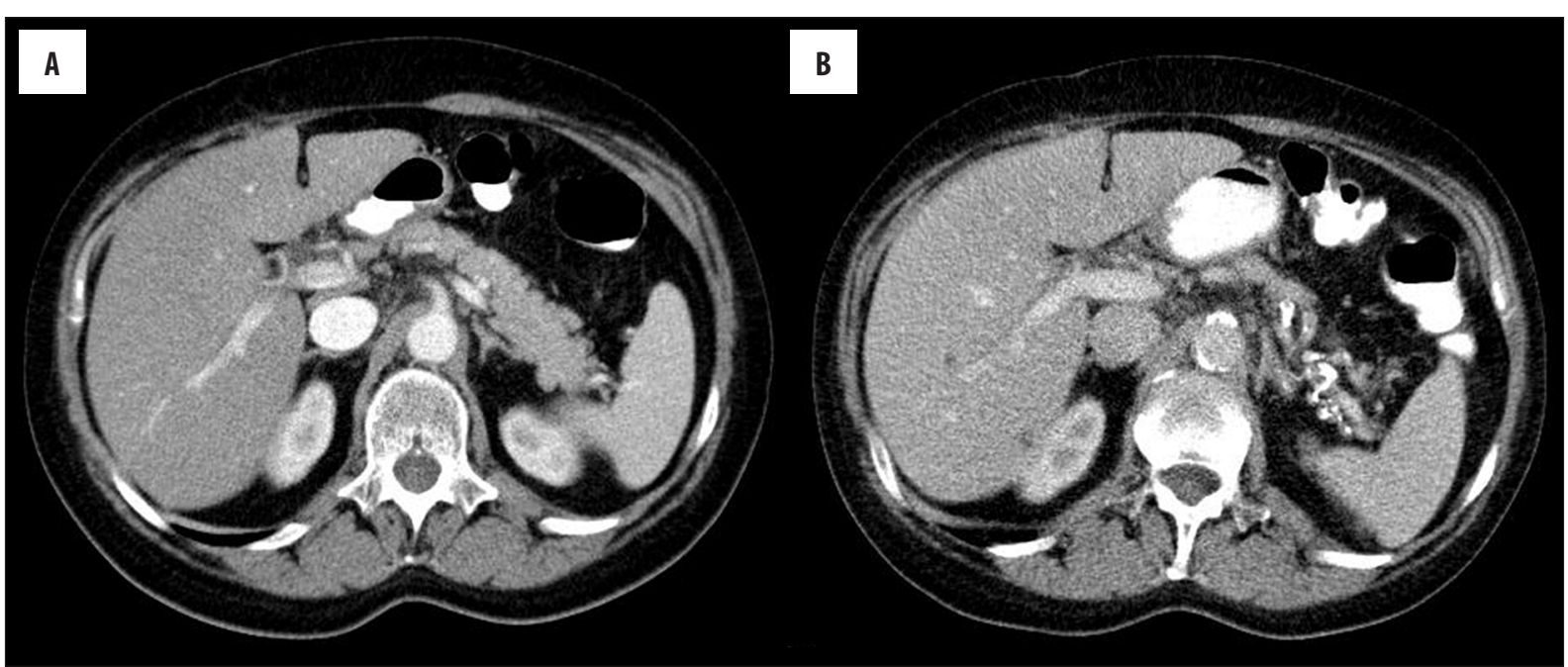

Figure 3. Axial CT scans during the venous phase of dynamic study before (A) and during chemotherapy (B); the " new "lesions are discernible within VI segment of the liver.

which represents $20 \%$ of all images of good response to treatment. In $4 \%$ of patients, lesion size increased, in $16 \%$ "new" lesions appeared.

There was enlargment of metastases in the first control examination in another 2 patients from the analyzed group despite good response to treatment but it did not exceed $20 \%$ of the sum of largest lesion diameters, so it was not classified as a progression in RECIST scale.

In 9 patients appearance of "new" weakly attenuating lesions in the liver was found in the course of treatment. In 2 patients it was visible in the first control examinations.

In the rest of the patients such images were found in further examinations (some patients had no available control examinations in the first 4 months of treatment).

Figure 3 shows examples of pseudoprogression of neoplastic process - appearance of "new" lesions in the liver after a few months of treatment.

Figure 4 shows example of metastatic lesion size pseudoprogression in the liver after 2 months of treatment, with simultaneous decrease in attenuation in the venous phase of dynamic CT scan.

\section{Discussion}

In the analyzed group of primary tumors that underwent treatment (14 cases), dimensions of all tumors decreased, as well as their attenuation (86\%). Contrast enhancement of most of the lesions after treatment was low $(43 \%)$ or absent (21\%). The remaining 36\% still expressed significant contrast enhancement, but it was lower than in baseline examinations, apart from two cases in which it remained stationary. In the course of treatment, the percentage of calcifications inside lesions increased from $29 \%$ to $36 \%$.

In the analyzed group of metastases and local recurrences (56 cases), similarly to primary tumors, most of the lesions shrank (75\%) and showed decreased attenuation in the course of treatment (79\%). As much as $46 \%$ of lesions did not enhance at all, and the remaining ones enhanced low or very low. The lesions, which held on to strong contrast enhancement did not response to treatment (18\%) or responded only by decreasing their dimensions (4\%). Contours of lesions became well defined in $39 \%$ of cases (11\% of cases before treatment). A certain group of metastases developed calcifications in the course of treatment $(4 \%)$. Features of temporary hemorrhage were more frequently seen after treatment (12.5\%) than before (4\%). In two cases lesions enlarged more than $20 \%$ from the starting point with simultaneously decreased attenuation. In another two cases lesions enlarged but less than $20 \%$.

In total, this constitutes 4 cases of false lack of response to treatment according to RECIST criteria, of which two would be classified as a progression, despite good response to drug. Therefore, to evaluate tumors after applied treatment, the Choi classification was introduced, according to which the lesion positively responding to treatment is the one, that decreases its dimensions by at least $10 \%$ or when contrast enhancement decrease inside the lesion is $15 \%$ or more.

Decrease in attenuation and size of neoplastic lesions in the analyzed group of lesions well responding to treatment during the whole observation period was largest during the first months of observation. In the third month after drug administration, median decrease in enhancement was about $40 \%$, and median decrease in size about $20 \%$. Both of those values were still decreasing in further observation, but not significantly.

In the group of 44 tumors investigated by Choi et al. in 2007, lesion attenuation after two months of therapy decreased by $31 \%$ on average in PR group, and lesion dimensions decreased by $26 \%$ on average. Those values were very similar to the ones in the analyzed group [6].

Betchold et al. noticed an average decrease in lesion attenuation of $47 \%$ in the second month of treatment (60 to 32 $\mathrm{HU})$, and of $62 \%$ in the twelfth month [7]. 


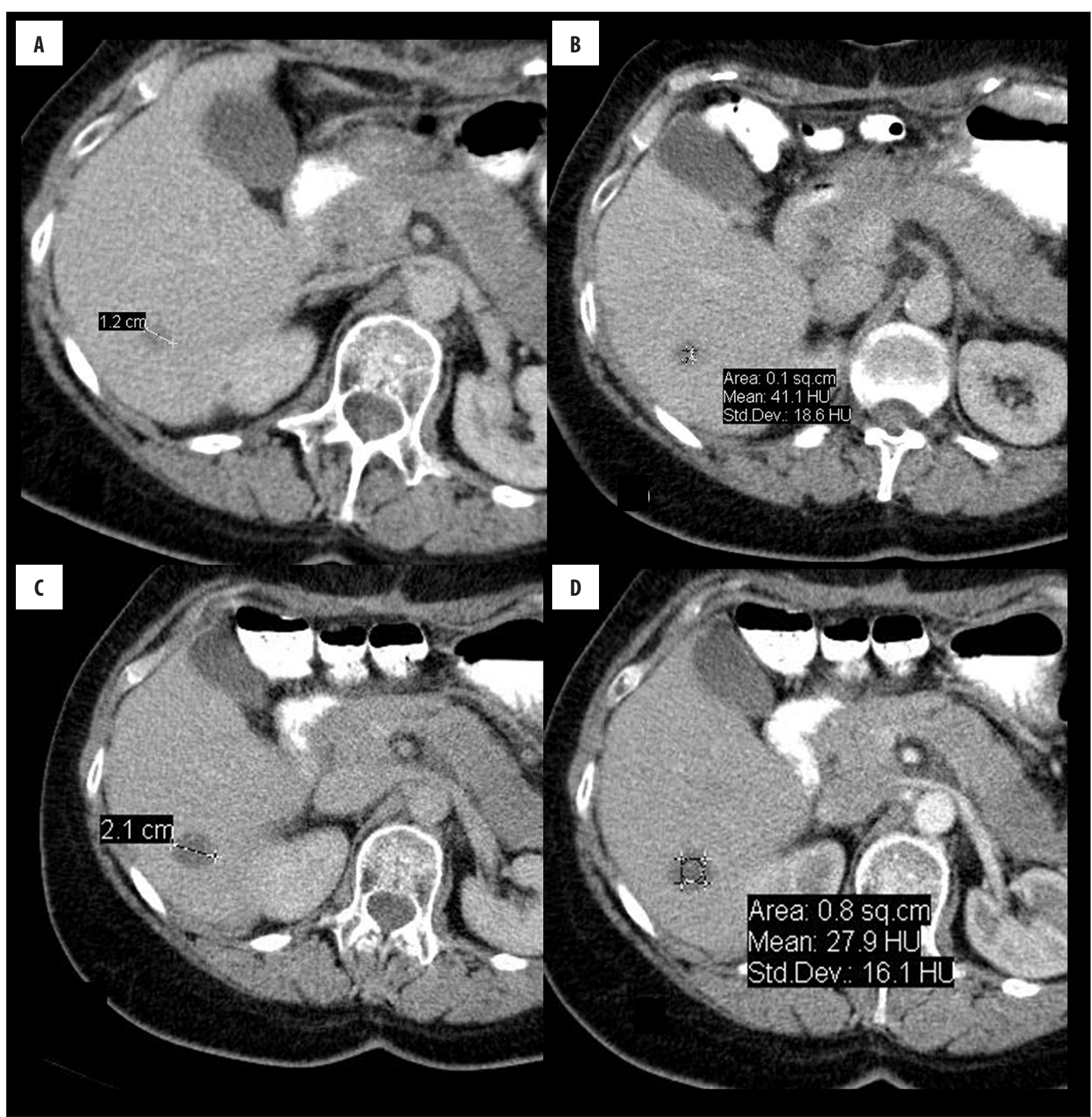

Figure 4. Axial CT scans in the venous phase of dynamic study of the metastatic lesion in the liver before (A, B) and after 2 months of therapy with Imatinib (C, D).The dimensions of the lesion increased from $12 \mathrm{~mm}$ to $21 \mathrm{~mm}$ (linear dimension), while the density of the lesion decreased from $41 \mathrm{HU}$ to $28 \mathrm{HU}$ (ROI).

Similarly, Sandrasegaran et al. reported that most significant decrease in size of lesions took place during the first 3 months of treatment [8].

The most common unconventional tomographic image, which can be the reason for diagnostic mistakes in evaluation of GIST treated with Imatinib, is the cystic transformation.

In the analyzed group of lesions, half of metastases in the course of treatment converted into lesions mimicking cysts, both in the liver and peritoneum. Those lesions did not enhance or had minimal contrast enhancement, had welldefined contours and interior attenuation values typical for liquids. The level of similarity between metastases and simple cysts was sometimes so high that wrong descriptions of such lesions in CT scans occurred (most often this referred to hepatic simple cysts and one mesenteric cyst), mostly in cases when there was no data on the diagnosis and treatment method in the referral letter. There was one case of interpreting fluidized metastasis in the pelvis as a free fluid in the peritoneal fold, with complete remission of lesions after treatment.

In case of metastases located in the peritoneum, detection and evaluation of lesions with cystic transformation, among liquid-filled bowel loops, is difficult. Proper filling of bowel loops with positive contrast agent can reduce these difficulties. What is more, due to mobility of the mesentery, metastases can migrate inside the abdominal cavity 
and pelvis; changes in their shape are also possible, which causes additional difficulties in evaluation.

Transformation of primary solid metastases in the liver into lesions mimicking simple cysts due to myxoid degeneration of neoplasm is a frequent phenomenon and was described in many articles [7,9-13].

Usually, attenuation of such lesions is a bit higher than of true cysts, which helps in differentiation [14]. Nevertheless, in the analyzed group we observed tumors with attenuation of a few to a dozen of HU, making them impossible to distinguish from real cysts based on the attenuation values only.

Sadragesian et al. found that $67 \%$ of hepatic metastases after three months of treatment looked like non-enhancing cystic lesions without internal echoes, and those lesions were often mistakenly described by radiologists as simple cysts [8].

Yang et al. described transformation of metastases into pseudocysts with clearly discernible contours of lesions, which had features of simple cysts also in ultrasonography, additionally increasing the chance of misinterpretation [15]. Similar transformations of solid tumors into pseudocystic lesions in CT scans were shown by Patnaik et al. [16].

Additional aspect of fluidization of metastases is the fact that according to RECIST criteria cystic lesions are considered to be "unmeasurable", and should not constitute targets measured in order to evaluate response to treatment, because changes in size of cysts do not necessarily correlate with the effect of the drug. The problem is serious because most of the GIST metastases undergo necrosis and cystic degeneration in the course of treatment. Usually in such cases there are no lesions that would remain solid in the course of treatment and whose changes in size could be followed according to RECIST criteria.

Difficulties described above do not occur when treatment response is evaluated according to Choi criteria, because decrease in attenuation occurring during fluidization of the lesions is the criterion of good response to treatment, regardless of the changes in their size.

Some authors suggest update of the criterion used by Choi, considering partial response as a relative decrease by at least $15 \%$ of lesion's attenuation, by adding a criterion of unconditional decrease by at least 10 Hounsfield Units [17].

Another unconventional tomographic image that can become the reason of diagnostic errors in evaluation of GIST tumors treated with Imatinib is enlargement of lesions during actual regression of disease (pseudoprogression). Such an image is considered in RECIST as progression of the disease. There is no threat of false interpretation of such lesions when Choi criteria are used because all the "growing" lesions that in fact response well to the therapy decrease their attenuation.

In the analyzed group, there were in total 4 cases of lesion enlargement accompanying good response to treatment, which resulted in qualifying two of them, according to the RECIST scale, to the group of patients with progression ( $4 \%$ of all patients), and the remaining two to the group with a stable disease, because lesion enlargement amounted for less than $20 \%$. In all of those cases, attenuation measured during the venous phase of the CT scan decreased significantly, especially in case of hepatic lesions that developed the image of pseudocysts (3 cases). All those lesions decreased in the second control examination, apart from one lesion, which remained stationary.

Rare cases of enlargement of tumors treated with Imatinib, despite good response to therapy (caused by rapid myxoid degeneration, necrosis or hemorrhage into the lesions), are described in papers, and the time that elapsed before the lesions stopped to enlarge was usually about 4-8 weeks (1-2 cycles of chemotherapy) [11,15,10,18-20].

Another unconventional CT image that can cause diagnostic errors in evaluation of GIST tumors treated with Imatinib is appearance of "new" changes suggesting disease progression during actual regression (pseudoprogression). It is caused by the fact that previously isodense, usually small metastases in the liver that decreased their attenuation in the course of treatment get visible, and therefore become detectable.

In the analyzed group of lesions such images of appearing "new" metastatic lesions in the liver were found in as many as $16 \%$ of treated tumors. In two cases it occurred already in the first control examination and resulted in qualifying the patients to group with progression (PD) based on RECIST criteria. In the remaining patients this was discernible in scans performed later than 3 months from the beginning of the treatment. All those new lesions had low internal attenuation and resembled in some degree tiny cysts in the liver. Sometimes such images were noticeable periodically because tiny hypodense lesions were becoming isodense with liver tissue or were decreasing so much that they could not be visualized anymore. Images of similar pseudoprogression probably occur in case of peritoneal metastases but were not seen due to difficulties with their noticing.

Descriptions of similar, rare cases of appearance of pseudonew lesions in the liver are also published in papers $[10,12]$.

Due to appearance of images of pseudoprogression (size and number) in the course of Imatinib GIST treatment it would be difficult to agree with conclusions made by Le Cesne et al. who claimed that the criterion of lack of progression according to RECIST classification is sufficient to distinguish patients with good response to treatment [21]

According to RECIST, patients with a false image of progression would be included in the group with disease progression (PD). Therefore, the criterion of alterations in tumor attenuation in the course of treatment cannot be omitted.

Tomographic images that could lead to diagnostic errors in the assessment of GIST tumors treated with Imatinib include temporary "disappearance" of metastases in CT 
scans as a result of diminishing their primary high attenuation, which then becomes isodense with the surrounding hepatic tissue. In the analyzed group of changes, temporary isodensity of metastatic lesions was present in as much as $23 \%$ of cases. This more often concerned small, single (or few) lesions in the same patient, who had remaining metastatic lesions visible anyway. Only in one case, all of the primarily well-enhancing lesions decreased their enhancement in the course of treatment, becoming isodense with the hepatic tissue for the period of a few months, so that it was difficult to measure their size and attenuation without referring to images prior injection of contrast agent, in which they were still (although weakly) visible. Such images can lead to qualifying the patient to the group with complete remission (CR).

In the analyzed group, we observed that metastases behaved in different ways in the course of treatment. Several patients had typical pseudocystic, non-enhancing lesions in the liver, coexisting with lesions with persistent (although decreased) contrast enhancement (3). What is more, in control examinations after a few to dozen or so months of treatment, we observed single cases of changes of different degree in the size of metastases in the same patient, as well as shrinkage of the lesions in the liver with simultaneous slow growth of the lesions in the peritoneum, or simultaneous shrinkage of some metastases with enlargement of the others in the same location (peritoneum or liver). Cases described above pose difficulties in radiologic evaluation of the treatment effect.

Phongkitkarun et al. found among 18 treated patients one case in which they claimed growth of lesions in the peritoneum, with lesions in the liver converting into cystic lesions that reduced their size [22].

Changes in the size of one of primary tumors were another problem in the analyzed group of GIST tumors. This was patient's only visible neoplastic lesion, which periodically evacuated its necrotic masses into the jejunum, thus changing its size inadequately to actual response to treatment of such tumor tissues. Based on own experience, we advise measuring wall thickness instead of total dimensions.

Tomographic image which can become a cause of diagnostic errors in GIST lesions treated with Imatinib is a stationary image of lesions during actual progression. Primary lesions with inner clonal expansion of cells that have lost sensitivity to drug - "tumors within a tumor" images usually retain their external dimensions. This has been described in many papers $[1,6,18,23-25]$.

In the analyzed group of GISTs there was a frequent phenomenon of stationary size of lesions in which progression type of "tumor within tumor" occurred (53\%), but also often pseudocystic lesions were decreasing during progression, although usually not significantly (42\%).

\section{Conclusions}

Since Imatinib was introduced to therapy of inoperative and disseminated GIST, CT and MRI have started to produce unique images, absent or very rarely encountered in conventional, cytotoxic chemotherapies used in other types of cancers. The possibility of their occurrence in the course of GIST treatment may lead to dangerous diagnostic mistakes and changes in the right treatment.

\section{Most common are:}

Tumor tissue "fluidization" image. Decrease in density and contrast enhancement level may cause transformation of primarily solid metastatic tumors in the liver into lesions mimicking simple cysts.

Pseudoprogression images. "Appearance of new metastases" in the liver during actual disease regression, caused by visualization of lesions whose contrast enhancement level prior treatment did not differ from the surrounding organ tissue. Such lesions can be completely invisible in the initial CT scan, especially when they are small, or get visible only in the arterial phase of dynamic study. After decrease in attenuation in the course of treatment, the lesions become visible and can be misinterpreted as progression, with dramatic effects on the patient.

Enlargement of metastatic lesions or their stationary size during actual disease regression are quite often encountered in the early period of chemotherapy. Enlargement of lesions, usually nonsignificant, can be caused by their abscess, hemorrhage inside tumor or myxoid transformation. In such cases, decrease in lesion density is the basic criterion of positive therapeutic response.

Stationary size of lesions during in course of local disease progression - a unique pattern of progression. Metastatic lesions, despite no changes in size (standard criterion of progression), especially with "cystic" features, can be a source of new clones of cancer cells, with genetic mutations determining drug resistance. It is expressed by appearance of tiny, better vascularized boundary nodes within maternal lesions. These nodes in their early stage can be easily overlooked by a radiologist. What is interesting, such new nodal hyperplasias are being observed also inside lesions without relation to the "cyst" wall, which proves that persistent, alive tumor cells are present also within lesions, suspended in the myxoid stroma. Some cancer cells surviving the course of therapy is the main problem in GIST chemotherapy which makes total cure impossible.

Diminishing of some metastatic lesions with concurrent enlargement of other lesions or development of new ones. Such images are caused by coexistence of metastases showing good response to drug, with new, resistant, expanding cell clones. This fact is of uttermost importance for radiologists evaluating an examination, as every metastatic lesion, regardless the other ones, has to be considered as a potential source of recurrence.

Due to a unique CT image of neoplastic lesions in the course of GIST during chemotherapeutic treatment, evaluation of treatment response requires application of Choi classification instead of RECIST classification. According to Choi classification, neoplastic lesions responding well to treatment do not always have to decrease their dimensions, 
which is a necessary condition according to RECIST classification. The dimensions of lesions responding positively to therapy can remain stationary or even increase. Stationary size of lesions after treatment does not necessarily prove stability of the neoplastic process. Therefore, the only reliable criterion of positive response to treatment according to Choi classification is decrease in lesion attenuation, which is a proof of decrease in neoplastic tissue vascularization. In case of a lesion positively responding to treatment, attenuation decrease by $15 \%$ or more should be confirmed.

\section{References:}

1. Vernuccio F, Taibbi A, Picone D et al: Imaging of gastroinestinal stromal tumors. Anticancer Res, 2016; 36: 2639-48

2. Miettinen M, Lasota J: Gastrointestinal stromal tumors. Gastroenterol Clin North Am, 2013; 42: 399-415

3. Radford IR: Imatinib. Novartis. Curr Opin Investig Drugs, 2002; 3(3): 492-99

4. Verweij J, van Oosterom A, Blay JY et al: Imatinib mesylate (STI571 Glivec, Gleevec) is an active agent for gastrointestinal stromal tumours, but does not yield responses in other soft-tissue sarcomas that are unselected for a molecular target. Results from an EORTC Soft Tissue and Bone Sarcoma Group phase II study. Eur J Cancer, 2003; 39(14): 2006-11

5. van Oosterom AT, Judson I, Verweij J et al: Safety and efficacy of imatinib (STI571) in metastatic gastrointestinal stromal tumours: A phase I study. Lancet, 2001; 358(9291): 1421-23

6. Choi H, Charnsangavej $\mathrm{C}$, Faria SC et al: Correlation of computed tomography and positron emission tomography in patients with metastatic gastrointestinal stromal tumor treated at a single institution with imatinib mesylate: Proposal of new computed tomography response criteria. J Clin Oncol, 2007; 25(13): 1753-59

7. Bechtold RE, Chen MY, Stanton CA et al: Cystic changes in hepatic and peritoneal metastases from gastrointestinal stromal tumors treated with Gleevec. Abdom Imaging, 2003; 28(6): 808-14

8. Sandrasegaran K, Rajesh A, Rushing DA et al: Gastrointestinal stromal tumors: CT and MRI findings. Eur Radiol, 2005; 15(7): 1407-14

9. Chen MY, Bechtold RE, Savage PD: Cystic changes in hepatic metastases from gastrointestinal stromal tumors (GISTs) treated with Gleevec (imatinib mesylate). Am J Roentgenol, 2002; 179(4): 1059-62

10. Linton KM, Taylor MB, Radford JA: Response evaluation in gastrointestinal stromal tumours treated with imatinib: Misdiagnosis of disease progression on CT due to cystic change in liver metastases. Br J Radiol, 2006; 79(944): e40-44

11. Choi H, Charnsangavej $\mathrm{C}$, de Castro Faria $\mathrm{S}$ et al: CT evaluation of the response of gastrointestinal stromal tumors after imatinib mesylate treatment: A quantitative analysis correlated with FDG PET findings. Am J Roentgenol, 2004; 183(6): 1619-28

12. Vanel D, Albiter M, Shapeero L et al: Role of computed tomography in the follow-up of hepatic and peritoneal metastases of GIST under imatinib mesylate treatment: A prospective study of 54 patients. Eur J Radiol, 2005; 54(1): 118-23
13. Gong JS, Zuo M, Yang P et al: Value of CT in the diagnosis and follow-up of gastrointestinal stromal tumors. Clin Imaging, 2008; 32(3): 172-77

14. Bechtold RE, Chen MY, Stanton CA et al: Cystic changes in hepatic and peritoneal metastases from gastrointestinal stromal tumors treated with Gleevec. Abdom Imaging, 2003; 28(6): 808-14

15. Yang DM, Kim H, Kang JH et al: Computed tomography and sonographic findings of hepatic metastases from gastrointestinal stromal tumors after chemotherapy. J Comput Assist Tomogr, 2005; 29(5): 592-95

16. Patnaik S, Jyotsnarani Y, Rammurti S: Radiological features of metastatic gastrointestinal stromal tumors. J Clin Imaging Sci, 2012; 2: 43

17. Kalkmann J, Zeile M, Antoch G et al: Consensus report on the radiological management of patients. Cancer Imaging, 2012; 12 126-35

18. Benjamin RS, Choi H, Macapinlac HA et al: We should desist using RECIST, at least in GIST. J Clin Oncol, 2007; 25(13): 1760-64

19 Diederich S: Imaging beyond RECIST: CT and MRI in molecular therapies. Cancer Imaging, 2012; 12(2): 347-50

20. Shinagare $A B$, Jogannathan JP, Krajewski KM: Liver metastasis in era of molecular targeted therapy new faces of treatment response. Am J Roentgenol, 2013; 201(1): W15-28

21. Le Cesne A, Van Glabbeke M, Verweij J et al: Absence of progression as assessed by response evaluation criteria in solid tumors predicts survival in advanced GI stromal tumors treated with imatinib mesylate: The intergroup EORTC-ISG-AGITG phase III trial. J Clin Oncol, 2009; 27(24): 3969-74

22. Phongkitkarun S, Phaisanphrukkun C, Jatchavala J, Sirachainan E: Assessment of gastrointestinal stromal tumors with computed tomography following treatment with imatinib mesylate. World $J$ Gastroenterol, 2008; 14(6): 892-98

23. Desai J, Shankar S, Heinrich MC et al: Clonal evolution of resistance to imatinib in patients with metastatic gastrointestinal stromal tumors. Clin Cancer Res, 2007; 13(18 Pt 1): 5398-405

24. Shankar S, vanSonnenberg E, Desai J et al: Gastrointestinal stromal tumor: New nodule-within-a-mass pattern of recurrence after partial response to imatinib mesylate. Radiology, 2005; 235(3): 892-98

25. Werewka-Maczuga A, Osiński T, Chrzan R et al: Characteristics of computed tomography imaging of gastrointestinal stromal tumour and related diagnostic problems. Pol J Radiol, 2011; 76(3): 40-51 